\title{
New partnership stands to save millions of mothers and children
}

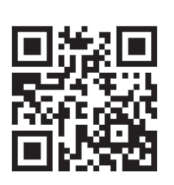

Two of several health innovations that could save the lives of millions of mothers and babies will probably reach the South African

(SA) market first - years ahead of schedule - because of a new partnership between the government, the South African Medical Research Council (MRC) and a leading international NGO.

With postpartum haemorrhage the leading cause of maternal mortality worldwide, the first innovation is a breathtakingly simple improvement on the first-line preventive medicine currently in use - the heat-sensitive injectable oxytocin. The intended product, a pill formulation, solves the longstanding dilemma of storage and supply chain management of liquid oxytocin in rural and/ or under-resourced areas, where most of the deaths occur. Not only will the prospective heat-stable 'under-the-tongue' fast-dissolving oxytocin pill be easier to store and manage, but less-skilled healthcare workers will be able to administer it, enabling quicker, better and more accessible prevention and treatment all round. The fixed-dose presentation has proved highly successful in animal studies and can withstand temperatures of up to $40^{\circ} \mathrm{C}$ for several months, making it ideal for tropical and subtropical climates, where most maternal deaths occur.

\section{Cutting R\&D-to-market} time by two-thirds

Developed by PATH, international non-profit leader in global health innovation, this is but one example of how products and devices can benefit from a health innovation accelerator initiative and partnership. PATH has now teamed up with SA's Department of Science and Technology and the MRC to apply the same booster principles locally, prioritising key gaps in maternal and infant health. PATH are specialists in accelerating innovation for vaccines, drugs, diagnostics, devices, systems and services, typically applying their knowledge and channelling funding to cut research and development-to-market time by up to twothirds. On average, it takes up to 15 years for a product to reach the market. SA's track record and successes in these fields make it the ideal partner, enabling PATH to accelerate clinical trials and regulatory approval and bring products to scale far sooner than would otherwise have been the case.

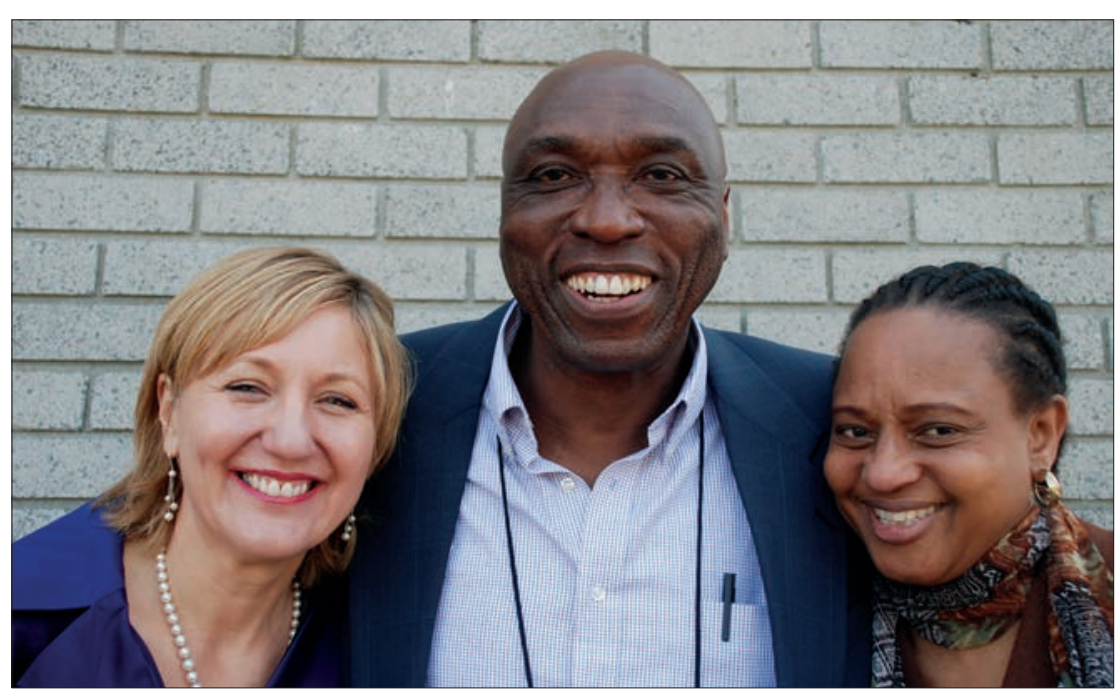

MRC President Prof. Glenda Gray, Dr Ayo Ajayi, PATH's Vice-President of International Development, and Dr Nonhlanhla Dlamini, Chief Director of Child, Adolescent and School Health, NDoH. Picture: Chris Bateman.

One local example of why PATH has quietly invested over R3.5 million in MRC activities over the past 3 years is a Stellenbosch University medical school-developed device called the 'Umbiflow', a mobile and highly versatile Doppler waveform analyser measuring placental insufficiency in small-for-gestational-age babies in the third trimester. In MRC President Prof. Glenda Gray's estimation, this would be the second of the two accelerated innovations to hit the local market first. Priceless in enabling early identification of potential birth problems and almost certain to generate major savings by avoiding unnecessary upward referrals, the Umbiflow was developed by the MRC and the Council for Scientific and Industrial Research (CSIR) under a grant from the former Innovation Fund, a Department of Science and Technologyfunded vehicle established to support local innovation. The machine detects blood flow within the umbilical cord, ascertaining placental function via a hand-held probe (similar to a sonar scan), but is connected with a USB cable to any Windows-based computer. By installing the necessary software, an integrated $3 \mathrm{G}$ card enables a mobile internet connection and automatic upload of exam results to a central server for remote expert support and electronic health record management. PATH will help accelerate the project even further, supporting continuing evaluation and subsequent implementation in antenatal facilities country-wide, with potential adoption by health systems across the African continent.
Leading by example with life-changing products and meds

Speaking at the launch of the MRC-PATH Global Health Innovation Accelerator (GHIA), Dr Ayo Ajayi, PATH's vice-president of international development, lauded SA's culture of innovative research dating back to Chris Barnard's first human-to-human heart transplant in 1967. He summed up PATH's mission as 'to get the best ideas and solutions and to translate and implement them where they can do the most good', taking innovations to scale to tackle the greatest health needs. He said that SA's deep expertise in vaccine development, drugs and devices made it the ideal partner to 'add the multiplier effect' by doing what PATH did best: getting involved in the middle stages of development, or the value chain. Globally PATH had reached 290 million people through various technological innovations so far, one of the most impressive being a meningitis A vaccine to which 153 million people across the 26 African countries where the disease is prevalent have had access over the past 4 years - its Indian manufacturer willing and able to churn it out for under 50 cents a dose. With the help of the World Health Organization, the vaccine was produced at one-tenth of the 'normal' research and development costs-to-market. Another notable PATH success story is the vaccine vial monitor, a tiny sticker put on vials of vaccine that changes colour when exposed 
to heat. Healthcare workers can tell at a glance whether heat-sensitive drugs are damaged. Ajayi said that more than five million stickers had been used worldwide so far.

Why we need life-saving innovation for mothers and babies

Speaking at the 8 August launch of the GHIA, Dr Nonhlanhla Dlamini, Chief Director of Child, Adolescent and School Health in the National Department of Health, illustrated just how badly needed effective new solutions are. She said that one-third of the country's under5 child mortality figures were due to just three newborn causes: prematurity, infection and birth asphyxia. 'If we can get a handle on these, we can overcome newborn deaths', she asserted. Broader under-5 mortality causes included diarrhoea, pneumonia, AIDSrelated illnesses and tuberculosis. She said that $65 \%$ of children who died in SA were malnourished, prompting the current African Union-funded infant and young child feeding policy. Recent innovations that were proving a huge success included the locally developed human papillomavirus vaccine, currently being administered with parental permission to all girls in grade 4 at state schools (3 000 SA women a year die of this cancer). The subdermal contraceptive implant was also being offered to girls who could legally opt to use it (from the age of 12). Of the one million women who fell pregnant in SA annually, $8 \%$ were girls below the age of 18 . These minors were giving birth to 80000 unplanned babies annually, contributing to $36 \%$ of al maternal deaths, with their youth contributing to fatalities. Dlamini said that other new vaccines added to the preventive arsenal (in 2009) were the rotavirus and pneumococcal vaccines. Their efficacy was vividly illustrated by the MRC's own rapid mortality survey this year, which showed a drop in the under-5 mortality rate from 56/1 000 children in 2009 to $41 / 1000$ in 2012 .

\section{Local products making global impact}

Dr Phil Mjwara, Director-General in the Department of Science and Technology, said among the projects currently being piloted was an earlywarning computer system for infectious diseases in Limpopo Province, including meningitis and water-borne diseases. Several companies had been born out of research efforts, including
Kapa Biosystems, which had developed reagents and kits for a number of DNA analysis applications such as real-time polymerase chain reaction (PCR) and high-fidelity PCR and molecular diagnostics. This company, which now exports its products, had expanded its local manufacturing base and research and development operations. Another major success story was Altis Biologics, which is on the verge of registering its regenerative biological implants as a fast and safe alternative to surgery for bone trauma patients. The injectable product was one of the most commercially viable and promising innovations he'd seen, winning the 2014 Innovation Prize Africa Award. Mjwara said that his department's collaboration with PATH and the MRC would 'benefit us tremendously as we seek to address the challenges of knowledge translation for South Africans'. The initial focus of the partnership would save 'millions' of vulnerable women and children in the country, he added.

\section{Chris Bateman}

chrisb@hmpg.co.za

S Afr Med J 2014;104(10):658-659.

DOI:10.7196/SAMJ.8887 